\title{
Further reflections on TOPKAT and Partial vs. Total Knee Replacement-response to authors
}

\author{
David J. Beard ${ }^{1}$, Andrew J. Price ${ }^{1}$, Marion K. Campbell ${ }^{2}$ \\ ${ }^{1}$ Nuffield Department of Orthopaedics, Rheumatology and Musculoskeletal Sciences, Botnar Research Centre, University of Oxford, Oxford, UK; \\ ${ }^{2}$ Health Services Research Unit, Health Sciences Building, University of Aberdeen, Aberdeen, UK \\ Correspondence to: Prof David J. Beard. Nuffield Department of Orthopaedics, Rheumatology and Musculoskeletal Sciences, Botnar Research Centre, \\ University of Oxford, Oxford OX3 7LD, UK. Email: david.beard@ndorms.ox.ac.uk. \\ Provenance and Peer Review: This article was commissioned and reviewed by the Academic Editor Dr. Ziming Chen (Centre for Orthopaedic \\ Translational Research, Medical School, University of Western Australia, Nedlands, Australia). \\ Response to: Carlson SW, Sierra RJ. Unicompartmental knee arthroplasty over total knee arthroplasty: a more cost-effective strategy for treating \\ medial compartment arthritis. Ann Transl Med 2020;8:510. \\ Argenson JNA, Jacquet C, Ollivier M. Medial femorotibial osteoarthritis of the knee: total or partial knee replacement? Ann Transl Med $2020 ; 8: 721$.
}

Submitted Mar 25, 2020. Accepted for publication May 09, 2020.

doi: $10.21037 /$ atm-2020-59

View this article at: http://dx.doi.org/10.21037/atm-2020-59

The acknowledgement by Argenson et al. (1) about establishing appropriate inclusion criteria for TOPKAT is appreciated and shows substantial insight. The potential for selection bias (particularly, but not exclusively, with cohort and non-randomised data) is ever present when considering comparative efficacy between two types of knee replacement. Even sophisticated post hoc adjustments and analyses can only account for, but never entirely obviate, such a critical and initial selection bias. Thresholds of disease and individual choice characteristics for implant type, by both surgeons and patients, are highly varied in normal practice and equipoise is often difficult to achieve (hence the Expertise design employed for TOPKAT) (2). Thus, the slightest bias, in an area which clearly lacks high effect sizes and enormous differences, will influence interpretation, conclusions (and therefore recommended practice). Using a randomised design in a carefully selected large population (suitable for both TKR and PKR) was a deliberate and key feature of the study and addresses some of the issues from other high quality cohort work $(3,4)$.

The issue of complications and revision rate is one of the more notable aspects of the findings, and again, very sensibly raised by all commentary authors. Admittedly, an RCT of limited sample is not ideal to detect the incidence of low frequency events, but the differences between RCT findings and registry data cannot go unnoticed. The likely explanation is that, despite the pragmatic design and external validity of multiple sites in TOPKAT, and unlike the surgeon body in any larger cohort or registry, none of the surgeons in the RCT were new to PKR. As such, registries (which will also include the performance of technically inexperienced surgeons) will undoubtedly influence the revision rate of a technically demanding procedure. Add in a likely lower threshold for revision outside the controlled environment of an RCT, and the inconsistency becomes less surprising. The main consequence of this contention is the disquiet surrounding recommendations for best practice. Until improved methodology is found future policy setting for practice must take the findings from both types of study into account. TOPKAT presently tells us that PKR in the hands of experienced surgeons is somewhat better, whereas registry data estimates somewhat higher rates of revision (5), most likely contributed from a less PKR experienced surgeon group and amplified by heterogeneity of revision thresholds in routine practice (although some assumptions are made here as we do not have that granularity of data). The conclusion, as was stated in the paper and in the commentaries, is to recommend PKR, but with a surgeon comfortable and experienced in the procedure (6). Note TOPKAT did not show any real detriment to TKR in comparison, and therefore surgeons who perform a good TKR for anteromedial OA should not be dissuaded from doing so. 
We also cannot disagree with Mssrs Argenson, Jacquet, Ollivier over their thoughtful final conclusion sentence and their point about 10 year data. A point reinforced by Carlson and Sierra from the Mayo Clinic (7) in their equally considerate commentary referencing their own quality study comparing PKR (UKA) and TKR in the older patient (8). Five year data is useful but the 10 -year picture will be more informative and we are in the process of collecting these longer term data. It will be less complete due to expected mortality but will still offer insight into the most appropriate long term surgical replacement for medial compartment OA. Indeed if there are larger or even different signals at 10 years we may have to revise our current interpretation. But that is exactly what constitutes good evaluation science - uncertainty, controlled bias, open mindedness and a justified conclusion.

However, as a postscript, merely obtaining 10 -year data will never guarantee inclusion in any "best practice" policy creation. Bias can take many forms and many external factors can affect acceptance and implementation of even the most robust science. For example, TOPKAT, despite its Lancet credentials and being the largest ever, mid-term, level-1 randomised comparison of PKR v TKR was outright rejected for peer reviewed conference and publication on several occasions. The interpretation of such action is much less straightforward...!

\section{Acknowledgments}

Funding: None.

\section{Footnote}

Conflicts of Interest: All authors have completed the ICMJE uniform disclosure form (available at http://dx.doi. org/10.21037/atm-2020-59). AJP reports personal fees from Consultancy with Zimmer Biomet, from null, outside the submitted work. The other authors have no conflicts of interest to declare.

Ethical statement: The authors are accountable for all aspects of the work in ensuring that questions related to the accuracy or integrity of any part of the work are appropriately investigated and resolved.

Open Access Statement: This is an Open Access article distributed in accordance with the Creative Commons Attribution-NonCommercial-NoDerivs 4.0 International
License (CC BY-NC-ND 4.0), which permits the noncommercial replication and distribution of the article with the strict proviso that no changes or edits are made and the original work is properly cited (including links to both the formal publication through the relevant DOI and the license). See: https://creativecommons.org/licenses/by-nc-nd/4.0/.

\section{References}

1. Argenson JNA, Jacquet C, Ollivier M. Medial femorotibial osteoarthritis of the knee: total or partial knee replacement? Ann Transl Med 2020;8:721.

2. Beard D, Price A, Cook J, et al. Total or Partial Knee Arthroplasty Trial - TOPKAT: study protocol for a randomised controlled trial. Trials 2013;14:292.

3. Liddle AD, Pandit H, Judge A, et al. Patient-reported outcomes after total and unicompartmental knee arthroplasty: a study of 14,076 matched patients from the National Joint Registry for England and Wales. Bone Joint J 2015;97-B:793-801.

4. Liddle AD, Judge A, Pandit H, et al. Adverse outcomes after total and unicompartmental knee replacement in 101,330 matched patients: a study of data from the National Joint Registry for England and Wales. Lancet 2014;384:1437-45.

5. National Joint Registry for England, Wales, Northern Ireland and the Isle of Man. 15th Annual Report. Available online: http://www.njrreports.org.uk/Portals/0/PDFdownloads/ NJR\%2015th\%20Annual\%20Report\%202018.pdf

6. Beard DJ, Davies LJ, Cook JA, et al. The clinical and cost-effectiveness of total versus partial knee replacement in patients with medial compartment osteoarthritis (TOPKAT): 5 -year outcomes of a randomised controlled trial. Lancet 2019;394:746-56.

7. Carlson SW, Sierra RJ. Unicompartmental knee arthroplasty over total knee arthroplasty: a more costeffective strategy for treating medial compartment arthritis. Ann Transl Med 2020;8:510.

8. Siman H, Kamath AF, Carrillo N, et al. Unicompartmental Knee Arthroplasty vs Total Knee Arthroplasty for Medial Compartment Arthritis in Patients Older Than 75 Years: Comparable Reoperation, Revision, and Complication Rates. J Arthroplasty 2017;32:1792-7.

Cite this article as: Beard DJ, Price AJ, Campbell MK. Further reflections on TOPKAT and Partial vs. Total Knee Replacement—response to authors. Ann Transl Med 2020;8(11):730. doi: 10.21037/atm-2020-59 